\title{
Configuración integral de las políticas públicas con énfasis social desde la actuación del trabajo social \\ (Setting public policies comprehensive social with emphasis from social work performance)
}

Fecha de recepción: 29 de noviembre-2013

Fecha de aceptación: 28 de febrero-2014

Yury Tatiana Mancera Agudelo

Universidad de Caldas

tatiamancera@gmail.com

\section{Resumen}

Citar políticas públicas, en noción de la necesidad social implica necesariamente activar la participación de trabajadores sociales y/o profesionales sociales en el cumplimiento de la pretensión del bienestar social.

Bajo esta idea y desde el reconocimiento teórico y referencial de las masas sociales, culturales a nivel latinoamericano ; la producción crítica-reflexiva en curso se focaliza la acción económica y productiva que aqueja a los diferentes grupos poblacionales; sobre el reconocimiento transversal del eje capitalista monetario en las dinámicas políticas, familiares, y humanas en la visibilización de una realidad social que requiere de un capital humano integral, este contrapuesto desde una justificación productiva del "norte social". Destacándose la óptica realy suicida que el trabajador social enfrenta en pro del ideal de ser parte de una mejor calidad de vida y en sí mejor bienestar de la condición humana con y desde la aplicabilidad de políticas públicas y sociales

\section{Palabras clave:}

Dignidad humana, ciudadanía, derechos humanos, política pública, política social, ética profesional.

\section{Abstract}

To quote public policies, taking into account the notion of social need, necessarily imply the involvement of social workers and/or social professionals pursuant to the provisions of social welfare.

Considering the fundamental idea which is developed in this project and from theoretical and referential recognition of cultural social masses throughout Latin America; the critical-reflexive ongoing economic and productive production is focused on what afflicts different population groups; about transversal acknowledgment of capitalist - money, in political, family and human dynamics, which is looking for a social reality that requires a comprehensive human capital, this contrasted from a productive justification "social north." Highlighting the real and suicidal optic in which social worker faces towards the ideal of being part of a better quality of life and human conditions and from the applicability of public and social policies

\section{Key Words:}

Human dignity, citizenship, human rights, public policy, social policy, professional ethics.

\section{ITINERARIOS DE INCLUSIÓN}


Configuración integral de las políticas públicas con énfasis social desde la actuación del trabajo social

(Setting public policies comprehensive social with emphasis from social work performance)

\section{Introducción}

Abordar la temática de políticas públicas, sin duda alguna, implica asumir compromisos conflictivos en términos de posiciones en extremo encontradas, una vez se focalizan elementos imperantes tales como condiciones reales de pobreza, exclusión social, invisibilización de los derechos humanos-dignidad humana, y adaptaciones "apropiadas" y particulares del sistema capitalista neoliberal convenientes para una parte mínima de la población, sea cual sea el contexto en donde se encuentra inmerso el y la trabajadora social. Éste panorama se precisa sobre la intervención profesional del perfil en curso particularmente, dadas las condiciones históricas y contemporáneas del ejercicio en términos de elementos asistencialistas, protectores, directamente relacionados con la jefatura estatal al idealizar el papel profesional como el hilo conductor que suple las necesidades básicas insatisfechas de las poblaciones y demás problemáticas sociales que agobian al capital humano adheridas principalmente por el factor económico.

Entonces, es insoslayable enunciar que condiciones de dinero y activantes de la economía como el capitalismo, equilibran y desequilibran al tiempo las condiciones sociales convirtiéndolas generalmente en tensiones al evidenciar el escenario de insatisfacciones, falta de accesibilidad a diferentes áreas como la educación, vivienda, salud, calidad de vida, incumplimiento de la proposición humana enmarcada en la dignidad humana que se caracterizan. Sin embargo, situaciones dificultosas como éstas emprenden el sentido ambiguo de la apertura a políticas públicas que intentan apostarle a abolir estados problemáticos y no fructíferos que tienen como rehén a la sociedad estancada. (Mancera, 20I I, p.3).

También vale la pena compenetrar el sentido de una política pública en conectividad con la referencia de una política social, que incluya en sí misma la adherencia de la participación ciudadana y colectiva en el trazo, diseño, alineación y ejecución de un sentir social enmarcado en el contexto estatal y/o gubernamental del que se depende.

En éste sentido, es de gran importancia la asociación y la mirada estatal-pública con énfasis directo en una política social, en tanto que se incorporan las necesidades y visiones de mejoramiento de las diversos componentes sociales como los ambientales, educacionales, familiares, laborales y de competencia, organizacionales, y culturales como garantía efectiva del cumplimiento de dicho sentir.

La línea de la política social para el entramado de realidades económicas y sociales se adscribiría así, en un ideal de eficacia pública y social donde tanto el componente estatal (estratégico) como el civil, se articulen en políticas públicas de carácter social: La política social consiste en situar a los ciudadanos en el núcleo de las políticas públicas, ya no mediante el suministro de asistencia social residual, sino incorporando sus necesidades y voz en todos los sectores. La política social es también instrumental, $y$ ha sido utilizada pragmáticamente por muchos gobiernos para conseguir el respaldo político de los ciudadanos, generar cohesión social, y potenciar un mayor desarrollo económico, mejorando el capital humano y el empleo productivo. La conexión con el desarrollo económico es particularmente importante. Las políticas sociales pueden superar el círculo vicioso de la pobreza y el atraso, y crear un círculo virtuoso en el que el desarrollo humano y el empleo generen una mayor demanda interna y crecimiento económico. (Ortiz, 2007, p.3)

\section{ITINERARIOS DE INCLUSIÓN}


Configuración integral de las políticas públicas con énfasis social desde la actuación del trabajo social

(Setting public policies comprehensive social with emphasis from social work performance)

\section{El conjugado de las políticas públicas: realidad económica y social como detonante de las políticas públicas}

Dada la pluralidad social (ésta entendida como las ideologías, construcciones y preferencias adaptativas de las personas) en la dimensión humana, frente a una mejor apuesta de desarrollo y evolución de sus mismas condiciones económicas, políticas, civiles, culturales, familiares y hasta personales, ha requerido pensar y repensar los escenarios modernos y postmodernos que han ido estructurando $y$ desestructurando las ideologías y actuaciones de los diversos referentes sociales en el apartado de bienestar, calidad de vida y, en general, de pretensión de equilibrio y eficacia integral de la subsistencia.

Así que en el sentido de subsistencia, articulado con factores económicos y de carácter rotatorio, éste como el contacto humano y social en contextos contemporáneos es preciso señalar, que dicha dinámica de adquirir "equilibrio" en todas las áreas humanas y sus recursos resulta estar inmersa en un juego de poderes "hegemónicos” que posibilitan y/ ó no dichas pretensiones. Un juego hegemónico, que se explicita sobre la plataforma de un capitalismo salvaje al evidenciar nociones excluyentes, inequitativas, degradantes y aberrantes contra el supuesto de desarrollo (conceptualizado sobre la evolución), pero acaso iserá sólo una evolución entendida como creciente y ascendente, que omite la vitalidad y el bienestar humano? (Mancera, 20I I,p.8)

Un juego dirigido por unos cuantos capitanes del mercado, que bajo sus directrices de auto productividad y autoritarismo han subordinado distintas zonas humanas vulnerables a la visión desalentadora de la pobreza, a la entrega de su mano de obra barata por la necesidad inmediata de recursos para la subsistencia y aumento mercantil del "poderoso", a la negación de una dignidad y condiciones "mínimas" de acceso a necesidades básicas, a una condición deplorable de su autoimagen y proyección, por cuanto el valor agregado de humanidad se desvanece con la realidad imperante de "necesidades" insatisfechas.

Una realidad, entonces enmarcada por enlaces bipolares como el tener y no tener capital, donde sin duda alguna, unos muy pocos tienen acceso efectivamente a un gran conjunto económico y también integral de las agencias sociales al incidir en el manejo y la "manipulación" del poco capital humano que queda.

Una ideología capitalista muy moderna y hasta postmoderna que evidencia intereses productivos y muy convenientes para ciertas corrientes de aplicabilidad económica y social y no tan convenientes para otros paradigmas activantes de la realidad social. Paradigmas y posiciones como el Keynesiano que, por su parte, reconoce la validación, presencia y emancipación del capitalismo en intereses de adquisición de recursos, acceso a la propiedad privada, a la inversión, al consumo pero con un ingrediente más y de respaldo o protector de posible inestabilidad y crisis de éste sistema como la participación del sector estatal, para referir una visión con lucros propios pero respaldada por papá estatal. Una visión en sí misma, que más allá de idealizar una garantía social (referencia humana) evidencia la preocupación por una estabilidad y manejo activo de recursos monetarios benéficos para la economía.

Por su parte y muy de la mano de Keynes, en el espacio del capitalismo, del cual hemos sido y aún seguimos siendo parte muchos expectantes se alterna Adam Smith, sobre su óptica liberal económica, la cual rescata la propiedad privada y su riqueza, el intercambio mercantil y productivo como garantía

\section{ITINERARIOS DE INCLUSIÓN}




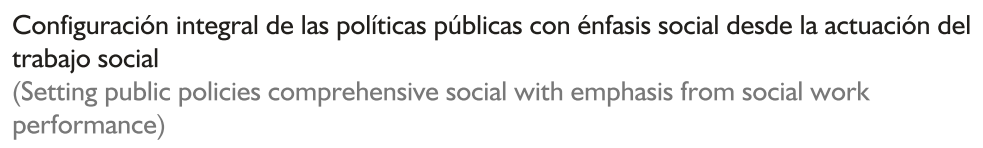

de una amplia accesibilidad y no delimitada de la adquisición y reproducción de los recursos que el Estado establece. Por su parte, éste ideal prefiere no diferir del mismo para la articulación económica más bien considera mucho mejor la ausencia estatal porque los ingresos y el propio capital se autorregulan a través de una "mano invisible".

Dichos referentes teóricos, para explicitar que a lo largo de nuestros tiempos las configuraciones económicas no solo han requerido del componente monetario como función en sí mismo de la adquisición de bienes y servicios, sino también, de una apuesta transversal social que directa e indirectamente es tocada por las diferentes apuestas económicas de la que es parte. Además, de la apuesta permanente por encontrar un "desarrollo integral" que parece alejarse de su objetivo intrínseco de mejoría para las condiciones del compacto humano.

Pero en este sentido, resultaría importante preguntarse isi acaso es acertado denominar "capital humano" sobre el escenario capitalista y neoliberal furioso que se centra básicamente en el aumento de la productividad material, la alianza macro empresarial, el enriquecimiento puntual de zonas sociales y la adquisición visionaria del monopolio?. Pareciera entonces que, el sentido inicial de propender por el desarrollo como una mirada de beneficio humano en todas las ópticas de acción y participación de los mismos, sobre un enriquecimiento humano integral se perdió en tanto factores predominantes como el enriquecimiento particularizado, la tecnología (acuñada a ingredientes modernos y postmodernos que "apartan" y hasta olvidan el sentir humano), la importancia material y el egoísmo social se entablaron fuertemente acabando con la libertad y el anhelo de "estar bien" de la sociedad.

Ahora bien, sobre esta conversación reflexiva y crítica, sería muy interesante traer a conversar los postulados de Amartya Sen (2004), sobre el enunciado principal de las libertades retenidas para la tergiversación del concepto de desarrollo y asociadas directamente con la visión económica como base de dicha dificultad. Una mirada social que rescata la necesidad de redistribuir equitativamente los recursos minimizando las condiciones de pobreza, la libertad de acceder a una serie de condiciones de naturaleza humana que le apueste a un desarrollo humano integral que se nutre de efectos ambientales, lucrativos, laborales, familiares, de habitabilidad (vivienda), educacional y, en sí mismo, de considerar un sujeto epicentrado que encuentra accesibilidad a la diversidad de su medio humano y en su amplitud social.

Toda la contextualización anterior, para efectuar de manera explícita el nombramiento y actuación de las políticas, como perspectiva de "inclusión" y mejorías de la cuestión social que se han nombrado en la configuración del papel hegemónico de las clases. Para esto entonces, según Roth A (20II) en su precisión de política pública enunciada que:

Se reserva la denominación de Política pública exclusivamente a las políticas (las estrategias y las acciones) de poder desarrolladas (directamente $u$ indirectamente, por acción u omisión) por la institución llamada Estado porque se considera que sus actividades corresponden a un interés público (o general) que le otorga legitimidad (principio de la democracia) a su actuar. (p. 9)

Un sentir entonces, consagrado directamente desde el ente estatal en prospectiva de cumplimiento con el amplio contenido de lo público, de "todos para "todos". Ahora bien, una vez entendidas las perspectivas sociales y materiales como acto complementario, es indispensable acotar que tales condiciones no terminan sus funciones en el simple reconocimiento, por el contrario, su continuidad abarca proposiciones de acciones reales que apuntan a la conceptualización de Políticas públicas como el ente regulador de las pretensiones colectivas y sociales de la ciudadanía.

\section{ITINERARIOS DE INCLUSIÓN}


Configuración integral de las políticas públicas con énfasis social desde la actuación del trabajo social

(Setting public policies comprehensive social with emphasis from social work performance)

Es decir, que detonantes (economía-sociedad) poderosos de la política pública, adscriben a la misma inicialmente un carácter estatutario, donde este poder legal y legitimizado establece facultades en términos de estrategias que propendan por la reducción de aspectos enmarcados en la pobreza, ajustes de la dignidad y derechos del hombre, bienestar social que incluya acceso a la salud y demás y en general evidencie el cubrimiento y protección de los ejes sociales y humanos que constituyen a una sociedad. Aunque con tales enunciados y discursos por efectivarse, la lucha y el reto son permanentes por el cumplimiento efectivo de todo lo citado.

Así, mientras se configuran las ideas por entender "cumplimiento efectivo de todo lo citado" se responsabilizan y se culpan "anónimamente" muchos silencios y atropellos que se han dejado pasar con el entorno y con el que hacer del trabajo social, de la ciudadanía y del ser humano en general cuando elementos de política pública sencillos como la salud, la educación y el respeto a la dignidad (en el marco de los derechos humanos), han sido violentados y la respuesta ha sido sólo la inconformidad gestual y lingüística. Sin embargo, hay una esperanza para asociar el carácter indispensable de lo social con lo estatal y gubernamental desde el actuar profesional del trabajo social, bajo el manto de la lucha y de los principios de ética y responsabilidad con nuestra gente además del enriquecimiento personal y espiritual por hacer llegar, acceder, promover y establecer razones y principios que garanticen una subsistencia saludable, aun cuando la inevitable mirada privada y capitalista exista.

Abordar las conjugaciones estatuarias en el concepto de política públicas, es reconocer que el motor innato de las mismas como las interacciones y cobertura social se ligan además a la óptica interdisciplinaria de lo social, en la cual indiscutiblemente aplica la intervención y cosmovisión de los y las trabajadores sociales en la construcción, seguimiento y ejecución de políticas sociales que acompañan el transcurrir estatutario.

\section{Perspectiva ética - ideológica del trabajo social en las políticas públicas ¿Obstáculo de una fachada incluyente con indirectas tóxicas?}

El ámbito de la perspectiva ética-ideológica del trabajo social, indica una serie de acotaciones normativas y al mismo tiempo ideológicas complementarias a un profesional pero también, a un ser humano, que a partir de sus diversos referentes culturales y demás, comparte ópticas convergentes y divergentes al respecto (Políticas públicas). Secuencialmente, se hace necesario precisar y contextualizar que el apartado ético, para el caso colombiano se fundamenta de la siguiente manera: :

La profesión de trabajo social requiere una sólida formación ética, epistemológica, política, teórica, investigativa y metodológica, para explicar y comprender científicamente la dinámica social, con el fin de implementar y gestionar políticas y promover procesos de participación y organización de la sociedad civil en la construcción y consolidación de la democracia. Consejo Nacional para la educación de Trabajo Social, [CONETS]_ (Ley I53 de 1977 decreto 2833 de 1981)

Alrededor del compacto ético, se introducen los grupos sociales, sus situaciones de vulnerabilidad y, en general, todo el trasfondo social que posibilitan la actuación profesional. Así mismo, la intervención se encuentra en permanente confrontación normativa (entendido como lo ético) y lo ideológico con las diferentes realidades en donde las decisiones prácticas más allá de un deber efectivo se convierten en dilemas de gran magnitud al exponerse frente a "tentaciones" nocivas tanto para su integridad

\section{ITINERARIOS DE INCLUSIÓN}




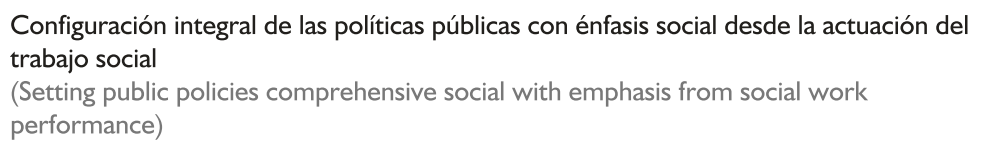

profesional como civil y personal conducidas al incumplimiento de nuestra ética. Si bien, se conocen los posibles obstáculos para el cumplimiento de los lineamientos éticos que nos competen, también es preciso enunciar que son los mismos apartados éticos e ideológicos los que nutren una perspectiva sólida del pensar, hacer y ser del trabajo social.

En éste caso y, del modo más preciso, los sujetos sociales cubiertos por el manto profesional se obligan durante su intervención a dar cuenta y cumplimiento de consignas éticas como: justicia, respeto, solidaridad, libertad, honestidad integralidad, transparencia, y confidencialidad; principios que retroalimentan una mirada científica-social pero que además se cristalizan con una óptica personal y civil, los cuales dan "conciencia" al tipo de realidad con que se enfrenta.

Entonces, evidenciada nuestra configuración ética no queda más que activar la misma mediante ejercicios propiciatorios de participación, inclusión y liberación de nuestras sociedades vulneradas a partir de estrategias sociales y de políticas sociales y no sólo queden en el filtro de las políticas públicas como el acceso de lo público y de todos, cuando ciertos ciudadanos pueden y tienen ventajas económicas y demás de otros y que en últimas como su nombre lo indica el sentido y el objetivo de la denominación "pública" de la política asocia a todo el conjunto social que le apuesten, verdaderamente, al logro de reformular dinámicas limitantes del desarrollo, progreso, bienestar y calidad de vida de los habitantes y en general de la sociedad. Las políticas sociales, además deben reconocerse como el esfuerzo por disipar las problemáticas causadas por el capitalismo y el neoliberalismo, indirectamente guardan otros esfuerzos o ayudas que potencialmente contribuirían a los "necesitados".

Sobre esta misma línea de consagración integral a la cobertura de bienestar y satisfacción social que le apuestan las políticas públicas además de la profesión de trabajo social, deben establecerse patrones claros, críticos y por supuesto éticos que confronten la corrupción y ansias de plusvalía de unos cuantos sucesores del cielo de la riqueza. No obstante, nuestra participación profesional, además de estar aliada con el cumplimiento y eficacia que, se supone, reproduce las políticas, debe estar preparada para contraponerse a miradas y ejecuciones perjudiciales para la población vulnerada. Contraponerse a políticas públicas sutiles que propician bajo su fachada la satisfacción de las necesidades sociales y no lo hacen, es determinar una intervención profesional impecable, transparente y comprometida con nuestras poblaciones en busca de mejores oportunidades. Del mismo modo no se desconoce el pensar y sentir que condiciones de carácter ético-ideológico en muchas ocasiones detienen circunstancias de beneficio personal, pero también que garantías simplistas y egoístas como éstas, no le pertenecen a profesionales adscritos al compromiso comunitario y social de los territorios a los que pertenecen y pertenecemos. Se concluirá relacionalmente con este aporte textual, que explicita el rechazo de actuaciones anti éticas, anti ideológicas y, en general, tóxicas para la cobertura humana que puedan llegar a propiciarse desde cualquier instancia, "Rechazamos un Trabajo Social incoloro, inodoro, e insípido” Ander, Egg pág. 26

\section{Actuación profesional en el campo de política pública asociada con el apartado social}

Este develar profesional, asociado con las prácticas y ejecuciones de políticas públicas, enraizadas al poder estatal, justifica una mirada integral en la que se desenvuelve no sólo bajo la cobertura de una relación institucional, sino bajo el compromiso social, ético-ideológico y de respeto con las comunidades y poblaciones que se integran al sistema social. De acuerdo a lo anterior:

\section{ITINERARIOS DE INCLUSIÓN}


Configuración integral de las políticas públicas con énfasis social desde la actuación del trabajo social

(Setting public policies comprehensive social with emphasis from social work performance)

“El Trabajo Social crítico, debe asumir la lectura reflexiva y cuestionadora de las dinámicas sociales, de las cuales como profesionales de la acción somos sujetos y objetos a la vez, lo que conlleva además a una crítica de nuestros propios marcos ideológicos con los que nos situamos en el mundo de la vida, que nos permiten aprehender, interpretar y darle significados las prácticas intersubjetivas, que van iluminando el quehacer profesional” (Vivero, 2010, p.147).

El trabajo social, desde sus múltiples escenarios de intervención, faculta y capacita al profesional sobre escenarios innatos a la sociedad ser determinantes, críticos y proactivos con las realidades problemáticas. De ahí, que se permee la estrecha brecha relacional de la profesión con la adscripción en el monitorio, diseño y ejecución de políticas sociales que le apuesten al objetivo focalizado del cubrimiento y efectividad de propiedades humanas como los derechos humanos, distribución equitativa de bienes -servicios y otros elementos de satisfacción social acuñados desde la óptica ética como un permeable importante.

El profesional, entonces, se somete a visualizar, leer y comprender la realidad contextual en la cual se encuentra inmerso para dar alternativas de solución ante dificultades de exclusión, de indiferencia, de marginalidad, de incumplimiento con los derechos adheribles a la esencia humana sobre el mecanismo de políticas sociales, las cuales facilitan el cumplimiento de la dinámica objetiva del trabajo social en cuanto se propone a ser mediadora, educadora y proactiva de los procesos sociales alcanzables de bienestar y calidad de vida para las poblaciones en las cuales interfiere. Nunca obviando, que la extensión y práctica profesional conlleva a confrontarse con dilemas de realidad, éticos e ideológicos frente al procesar e intentar cumplir lo "previsto". Cuán importante se convierte adscribir la profesión a procesos políticos, democráticos, y también sociales (entendido como las dinámicas humanas) a la propiciación de la plataforma de las políticas sociales, donde la perspectiva ética se conexione al acercamiento de ideales de calidad de vida. Inscritos en éste tipo de procesos, seguramente la actuación profesional arrojará acercamientos precisos de las necesidades por intervenir y además de las estrategias - para él, el vislumbramiento de soluciones efectivas y eficaces que suelen puntualizarse inicialmente sobre factores relevantes como la pobreza.

De ahí que reconocidos factores inherentes y transversales de la pobreza y sus acompañantes expresivos de exclusión e inequidad, anden tan tranquilamente por el espacio social dejando preocupaciones de cómo intervenir "adecuadamente" a los y las profesionales. Seguramente sobre esta posición, girarán miles de interrogantes por parte de los y las científicas sociales para abordar de una mejor manera la problemática sobre la cual se sostiene, tratando de encontrar metodologías, métodos, maneras y estrategias que se acercan al ideal, y así también, apoyarse de políticas sociales, para que más allá de estatutos y compromisos "socialmente" legitimados y legales, se efectivice la mirada de mejoramiento y cubrimiento de las necesidades insatisfechas que constituyen el objetivo presencial de dichas opciones estatales.

Finalmente:

La praxis profesional, entonces, se consolida como el resultado a partir del cual él y la Trabajadora Social requiere de un ejercicio de Auto revisión, a partir del cual la consolidación de avances estratégicos, que partan de soslayary entender lo social como aquel eje movilizador de sensaciones finalizan ideas en las que se sustenten situaciones de opulencia y penuria, lugar donde los ideales humanos constituyan la razón esencial por la cual es necesario movilizar y apropiar condiciones propositivas a partir de espacios de reflexión y visiones cortantes de realidad que se alejen de concepciones monopolizadoras, inútiles, en un todo autocráticas con la intención de hacer del actor social un sujeto capaz de pensarse a sí mismo, pensar en los demás (reconocimiento del

\section{ITINERARIOS DE INCLUSIÓN}


Configuración integral de las políticas públicas con énfasis social desde la actuación del trabajo social (Setting public policies comprehensive social with emphasis from social work performance)

otro), pensar en un nosotros, recuperando la perdida de solidaridades, en las que los ideales colectivos se visibilicen. (Manceray Oramas 20II).

\section{Conclusiones}

En el marco de intervención y praxis profesional del trabajo social, sin duda alguna, las políticas sociales configuran dicha actuación baja la mirada focalizada de incidir satisfactoria y eficazmente sobre los escenarios conflictivos que poseen las poblaciones y comunidades. Esto, como consecuencia de los efectos capitalistaneoliberales que someten además, a las dinámicas sociales a enfrentarse con desventajas de carácter económico, social, distributivo y demás.

Elementos como la democracia, la participación, la inclusión, el trabajo colectivo, las oportunidades, la distribución equitativa, la justicia, y el cumplimiento de los derechos humanos asociados con la plataforma ética y de intervención en la profesión deben permearse constantemente. Aunque una vez, la realidad se encargue de dictar otra referencia como la invisibilización de los mismos, es deber, desde la profesión del trabajo social, propiciarlos e intentar cumplirlos. Sobre todo por el marco normativo y ético que configura una intervención con intensiones saludables para la población o poblaciones a intervenir.

Frente a la apuesta de un desarrollo humano integral, comprendido ampliamente como la satisfacción económica, satisfacción de dignidad humana, satisfacción emocional-familiar y personal, satisfacción social y civil, y en sí satisfacción complementaria a las demás áreas del sujeto social se precisa señalar que el referente en mención se convierte en un anhelo ideológico con intención de efectivarse pero de difícil ejecución.

Por último, es preciso resaltar la importancia de un y una profesional comprometidos con el bienestar social, pero también con un compromiso personal e individual que refleje comportamientos de conciencia, de reflexión permanente por su cotidianidad, un sentido crítico en sus condiciones de reflejarse intrínseca y extrínsecamente. Profesionales que, además de perpetuar un "ideal social", reconozcan y evidencien desde sí mismos, el respeto y por qué no, como diría un proverbio, el amor de sí mismos por los demás en la lucha incansable que el trabajo social tiene por un mundo diferente. Finalmente, si se retrocede a la historia de nuestra profesión, se confirmará que el sentido inicial se establece sobre la transformación social.

\section{Referencias}

ANDER, Egg Ezequiel “ Metodologias y métodos del trabajo social” 2003

Del Sol, J "Resumen de desarrollo y libertad de Amartya Sen” 2004

KEYNES, John Maynard. Biografías y Vidas 2004

http://www.biografiasyvidas.com/biografia/k/keynes.htm

Mancera T. El apartado ético-ideológico del y la trabajadora social en las políticas sociales. En $4^{\circ}$. Congreso Internacional en Trabajo Social y Transdisciplinariedad en el siglo 21 . México

\section{ITINERARIOS DE INCLUSIÓN}


Configuración integral de las políticas públicas con énfasis social desde la actuación del trabajo social

(Setting public policies comprehensive social with emphasis from social work performance)

Mancera T. El sujeto epicéntrico como primera mirada del constructo social en la intervención del trabajo social. En $4^{\circ}$. Congreso Internacional de Trabajo Social. En el $8^{\circ}$. Congreso Nacional de Trabajadores Sociales de la Salud. En el $2^{\circ}$. Congreso Nacional e Internacional de Rehabilitación Social y Ocupacional. Cuba

Ortiz, I. (2007) Guías de orientación de políticas públicas-Política Social Organización de Naciones Unidas [ONU]Nueva York

Roth, A. Perspectivas teóricas para el análisis de las políticas públicas: ¿de la razón científica al arte retórico?" Artículo reflexión

Medellín, Colombia 2008

\section{SMITH, Adam "Vida y Obra” Enlaces latinoamericanos para economistas/ Biografías}

VARGAS, Mónica - MERCADO, Admundo "Política social y Trabajo Social un análisis histórico, desafíos, dilemas y propuestas" Ponencia

VIVERO, Luis A. "Hegemonía y lucha de clases en la práctica del trabajo social” Artículo crítico-reflexivo en Revista Eleuthera.

año 2010

\section{Notas}

I Articulo de reflexión crítica 